\title{
Follow-up of gastric cancer: a review
}

\author{
John Whiting ${ }^{1}$, Takeshi Sano ${ }^{2}$, Makoto Saka ${ }^{2}$, Takeo Fukagawa ${ }^{2}$, Hitoshi Katai ${ }^{2}$, and Mitsuru Sasako ${ }^{2}$ \\ ${ }^{1}$ University Hospital Birmingham, Birmingham, UK (Visiting fellow at the National Cancer Center Hospital) \\ ${ }^{2}$ Gastric Surgery Division, National Cancer Center Hospital, 5-1-1 Tsukiji, Chuo-ku, Tokyo 104-0045, Japan
}

\begin{abstract}
Although there is broad agreement in the staging, classification, and surgery for gastric cancer, there is no consensus regarding follow-up after gastrectomy. Follow-up varies from investigations on clinical suspicion of relapse to intensive investigations to detect recurrences early, assuming that this improves survival and quality of life. Advanced gastric cancers recur mainly by locoregional recurrence or distant metastasis. Local recurrences detected at endoscopy or on computed tomography (CT) are invariably incurable. For early gastric cancers, endoscopy can detect new primaries, but the incidence of these tumors is low, and many thousands of procedures are required to detect each operable case. CT is much better at detecting liver metastasis and, although these are usually multiple and unresectable, there are several reports of good survival following liver resection for isolated metastasis. Tumor markers have been used with some success to detect subclinical recurrences and could be used to target more invasive or expensive procedures. In chemotherapy, many newer agents are promising significantly improved survival, but again, the evidence for greater benefit when administered prior to the patient becoming symptomatic is lacking. Overall, it appears that follow-up policy is as much decided by the wealth and facilities of the institution as by any significant evidence base. Although the early detection of recurrent cancer is an emotive issue for both patients and surgeons, considering the amount of time and money invested in follow-up, and the lack of evidence of efficacy, a randomized controlled trial of intensive follow-up is required.
\end{abstract}

Key words Stomach neoplasms - Gastrectomy · Follow up · Recurrence

\section{Introduction}

Surgery for gastric cancer is becoming more successful. In the West, units with special interests in gastric cancer

Offprint requests to: $\mathrm{T}$. Sano

Received: December 26, 2005 / Accepted: January 6, 2006 have improved the proportion of cancers that are diagnosed at a potentially curative stage to $63 \%$ and are reporting 5-year survival rates of $70 \%$ for $\mathrm{R} 0$ resections $[1,2]$. The centralization of services, earlier diagnosis, and more successful surgery has greatly increased the number of patients requiring follow-up. In a unit performing $50 \mathrm{R} 0$ resections a year, approximately 150 patients will be undergoing follow-up at 5 years, and 200 at 10 years. In Japan and other countries in Eastern Asia where large units perform over 500 gastrectomies per year, with the majority for early-stage disease, the problems of follow-up are increased by at least an order of magnitude.

There are three main reasons for follow-up: to detect problems associated with the operation, to collect outcome data, and to detect recurrent disease. Many units actively investigate patients in order to detect recurrences at an earlier and asymptomatic stage, in the hope that this will lead to improved outcomes. The evidence for this, however, is weak and several surgeons have questioned the use of scarce resources in intensive follow-up.

In colorectal cancer, several randomized controlled trials (RCTs) and metaanalyses have demonstrated improved survival in patients undergoing intensive followup [3], and national bodies such as the American Society of Clinical Oncology (ASCO) and the Association of Coloproctology of Great Britain and Ireland have issued guidelines on the follow-up of colorectal cancer [4,5]. ASCO guidelines are also available for other cancers, such as breast [6] and lung [7], but for gastric cancer they are notable by their absence. Even the Japanese Gastric Cancer Association (JGCA) guidelines, which are proscriptive in the diagnosis and surgical treatment of gastric cancer, offer no guidance on follow-up [8]. This lack of guidance is unsurprising, given the paucity of high-quality evidence and the complete lack of RCTs. In the absence of national protocols, surgical units have adopted widely disparate regimes, 
and in a discussion of gastric cancer follow-up involving centers from the United States (Memorial SloanKettering [MSK], Roswell Park, and University of Washington), United Kingdom (Royal Liverpool), and Japan (National Cancer Center [NCC]) the only constant was visits to the outpatient department [9]. Routine endoscopy is performed by all centers, but not by MSK, on high-risk (pT3, pT4) patients, as recurrent disease in this group is rarely curable. Recommendations for routine computed tomography (CT) scanning ranged from not at all to biannually for 5 years, and there was little agreement on what tests were useful, how frequently they should be performed, or for how many years they should continue. Overall there is little consensus on an appropriate follow-up regime, and the efficacies of such programs remain ill documented. Although articles quote recurrence rates or patterns, most reveal little if anything about how recurrences are treated, and with what success [10-14].

This article aims to examine the literature and to present the evidence for the efficacy of follow-up protocols and investigations after gastrectomy, from the dual viewpoints of detecting and treating asymptomatic recurrent disease. It does not address follow-up of patients after endoscopic mucosal resection (EMR), as cancers treated by EMR have different recurrence patterns and outcomes $[15,16]$.

\section{Recurrence patterns of gastric cancer}

Gastric cancer has four broad patterns of recurrence: local recurrence either in the gastric bed or regional lymph nodes, peritoneal dissemination, liver metastasis and distant metastasis. In the West, the pattern of recurrence tends to be local. In the 1982, article by Gunderson and Sosin, ${ }^{17}$ examining recurrence patterns discovered at planned re-laparotomy following curative resection, 86 of 107 patients had recurrences, with 82 evaluated. Distant metastasis alone accounted for 21 (26\%) failures, but local failure was more frequent, present in $72(88 \%)$ patients. In $44(54 \%)$ patients, this was the only failure modality. In an Italian series with 215 recurrences from 441 gastrectomies, 96 (45\%) suffered local recurrence, 57 (27\%) hepatic, 77 (36\%) peritoneal, and $20(9 \%)$ distant metastases [10]. In the East, the pattern is different, with fewer local recurrences. In a series from Japan, of 939 operated patients, there were 207 recurrences, of which 130 had complete records. Recurrence was local in 29 (22\%), $56(43 \%)$ were peritoneal, 43 (33\%) hepatic, and $27(21 \%)$ distant; and $25 \%$ had recurrences at multiple sites [11]. In a large series from Korea examining 508 patients with recurrent cancer from an initial 2328 operated patients, 425 had recurrence at only one site, $98(23 \%)$ had local recurrence, 172 (40\%) had peritoneal recurrence, $75(18 \%)$ had hepatic secondaries, and $80(19 \%)$ had distant metastasis [18]. The lower local recurrence rates in the East appear to be related to the routine performance of D2 lymphadenectomy, as use of this technique in the West leads to similarly low local recurrence rates [14]. Risk factors for recurrence include greater stage of disease, undifferentiated (Lauren diffuse) type, and proximal tumors [18,19].

Early gastric cancer (EGC) carries a very favorable prognosis, and, in a report from the NCC Tokyo following up 1475 patients with EGC, only $1.4 \%$ were found to have recurrent disease. Of the 20 recurrences, 11 were at multiple sites, 1 was peritoneal, 4 were local, and 4 were hematogenous [20]. A similarly low rate was reported from 1452 patients with EGC from Korea, with only 4 local recurrences and 2 recurrences confined to the liver [21].

It is worthwhile to be aware of the time scale over which disease recurs. The consensus appears to be that over two-thirds of recurrences occur in the first 3 years and that fewer than $10 \%$ occur after 5 years $[12,19,22]$. In EGC, there is a difference in the reported literature regarding recurrence times, with Lee et al. [21] reporting the majority $(62 \%)$ of recurrences detected at less than 2 years and fewer than $10 \%$ occurring after 5 years, while Sano et al. [20] reported deaths from recurrences occurring later: $23 \%$ occurring after 5 years and only $40 \%$ occurring within the first 3 years.

Adjuvant treatments after gastrectomy may alter patterns of recurrence. In the adjuvant chemoradiation study of MacDonald et al. [23], adjuvant treatment reduced the proportion of patients recorded as having local and regional recurrences as the first site of relapse from $29 \%$ and $72 \%$, respectively, in the surgery alone group to $19 \%$ and $65 \%$ in the chemoradiation group.

There is evidence that specific pathological features of the resected tumor can provide insights into the likely modalities of recurrence, allowing follow-up plans to be modified. For example, for T1/2 N0 tumors with histological evidence of venous capillary infiltration, recurrence is invariably by hepatic metastasis. Although bone secondaries are relatively uncommon, in poorly differentiated or signet-ring cell carcinomas with very extensive nodal involvement, they are more likely, and some may respond to chemotherapy [24].

\section{Second primaries}

Second primary cancers can arise in the remnant stomach and they can occasionally be difficult to differentiate 
from recurrence. Much of the literature relates to gastrectomies for peptic ulcer disease, and it was thought that the bile reflux that was common after Billroth I and II type operations caused an increase risk of gastric cancer after decades, and a meta-analysis, published in Cancer Research, estimated a relative risk of 1.48 after 15 years [25]. However, in a study of 6459 patients over 25 to 33 years, the rate of second primaries was not different from that in the general population [26]. After partial gastrectomy for cancer, the risk of second primaries appears small; in one Korean series of 1452 patients followed for EGC, only 5 such tumors were discovered, representing an approximate annual incidence of 70 per 100000 [21]. In the study of Kikuchi et al. [27], of followup endoscopy in 210 patients who underwent gastrectomy for EGC, after 6 years, 2 were found to have further gastric cancers, representing an annual incidence of 160 per 100000 . In addition, one esophageal cancer and one duodenal cancer were found, and importantly, all of these new cancers were treatable by endoscopic mucosal resection (EMR). Like primary gastric cancers, second primaries are amenable to surgery if detected early. In cases not suitable for EMR , R0 resection rates of up to $85 \%$ have been reported [28], with 5-year survival of approximately $40 \%$ [29-31].

\section{Detection of recurrent disease}

There are many investigations that may be used to detect recurrent gastric cancer, and these can broadly be divided into endoscopy, imaging, and blood tests. Endoscopy has the ability to detect intraluminal recurrences with a high degree of accuracy and it also has the ability to detect new cancers at a treatable stage.

The use of tumor markers has become more commonplace. Carcinoembryonic antigen (CEA) and Carbohydrate antigen (CA) 19-9 levels are easily determined by a simple blood test and have reported sensitivities of between $16 \%$ and $65 \%$ for individual markers, increasing to up to $85 \%$ if both were used [3234]. Increases in markers are commonly seen prior to the clinical detection of recurrences, and in a prospective study, both tumor markers were useful indicators of recurrence, even in patients whose original tumors did not express them $[34,35]$. Other tumor markers, such as CA 72-4 and CA 125, have been investigated, but sensitivities are significantly lower than those for CEA and CA19-9 [33].

Reports on the use of imaging in detecting recurrent gastric cancer are few, and are often limited to descriptions of typical findings $[35,36]$. The ability of imaging to detect recurrence is poor and this has been recognized in the design of trials of chemotherapy. For many cancers, disease-free survival, as documented by imaging, is a primary endpoint, but in an ongoing trial in gastric cancer, this endpoint has not been used because of poor accuracy [37]. The ability to detect hepatic metastases is probably also overestimated, and has been examined in a recent metaanalysis of trials comparing the accuracy of several imaging methods. When the required specificity was set at greater than $85 \%$, the most sensitive method was 18 F-fluorodeoxyglucose positron emission tomography (PET) with a sensitivity of $90 \%$, followed by magnetic resonance imaging (MRI; 76\%), CT (72\%), and ultrasonography (US; 55\%) [38]. In gastric cancer, the great majority of patients under follow-up will not have hepatic metastases, and even with high specificities, there are likely to be many false-positive results. The ability of CT to primarily diagnose a primary carcinoma of the stomach is not as good as its ability to stage a known cancer, and there is a direct tradeoff between sensitivity and specificity. In one report, when the criterion for diagnosis was a gastric wall thickness of $2 \mathrm{~cm}$ or more, sensitivity was only $50 \%$ and specificity $88 \%$. When the criterion was reduced to $1 \mathrm{~cm}$, sensitivity was $100 \%$, but specificity dropped to only $36 \%$ [39].

As well as inaccuracies in detecting local recurrences and hepatic metastasis, conventional imaging is poor at detecting peritoneal disease, which accounts for around $40 \%$ of recurrences. Barium enema has been described as useful in the diagnosis of such recurrence in colorectal cancer [40], and this method has been used at some Japanese institutes to confirm the presence of peritoneal disease suspected clinically (Fig. 1).

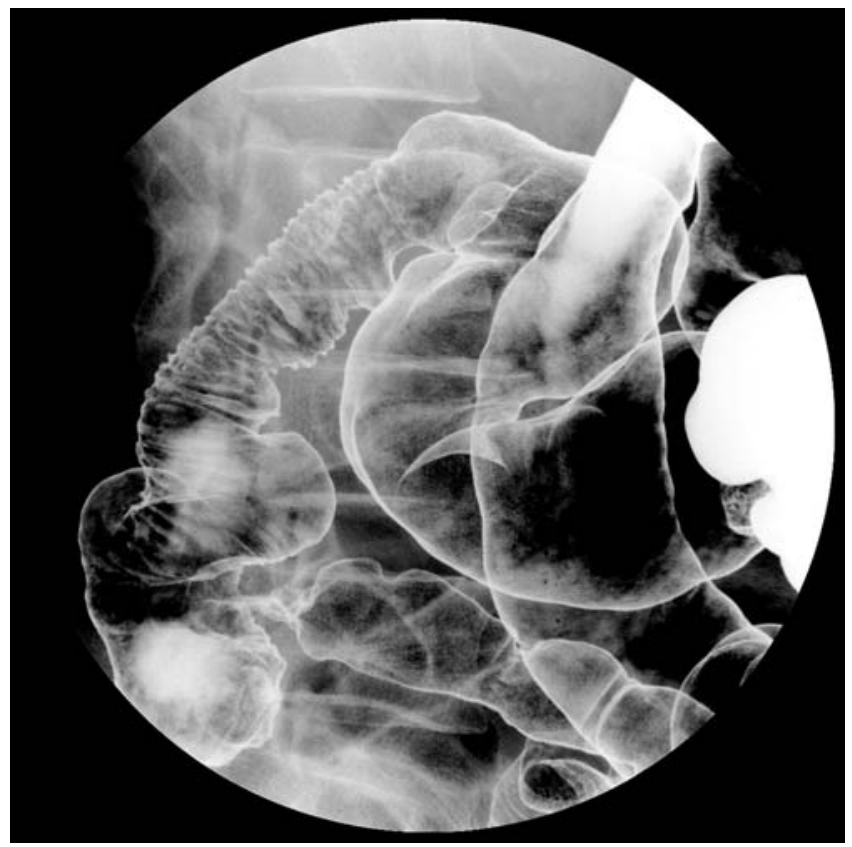

Fig. 1. Barium enema showing a typical "bellows" appearance caused by peritoneal dissemination 
From the previous discussion it can be appreciated that imaging in the search for asymptomatic recurrence is fraught with difficulties, missing many recurrences and producing a number of false-positive results. Imaging is perhaps more useful when a clinical recurrence is suspected, such as in the face of rising tumor markers. In this role, PET can be especially useful in cases where conventional imaging results are equivocal, as it can confirm or refute the presence of recurrence in most cases [41]. Overall, the ability to detect asymptomatic recurrences - despite intensive follow-up - is poor, with the proportion of such recurrence detected varying from only $22 \%$ to $45 \%$ [22,42].

\section{Treatment options}

\section{Surgery}

Peritoneal disease and distant metastases are not amenable to surgery with curative intent. Theoretically, local recurrence, if detected at an early stage, could be amenable to surgery, but this is not borne out in the literature, with only occasional reports of longer-term survival $[43,44]$. In a study of 197 recurrences, both symptomatic and asymptomatic, 41 surgical procedures resulted in no cures [22].

The treatment of liver metastasis has undergone a revolution. Traditionally seen as indicative of advanced, incurable disease, in colorectal cancer, hepatic resections for metastases are now routine, and 5-year survival rates of $40 \%$ are achieved $[45,46]$. The treatment of liver metastasis from upper gastrointestinal cancers is still seen by most surgeons as fuftile, but some evidence is now available that challenges this notion. In 1994, Ochiai et al. [47] reported on a retrospective series of 21 patients who had undergone liver resection for synchronous or metachronous gastric cancers, where 4 patients were alive 5 years post-resection. In 1997, Miyazaki et al. [48] reported on 21 similar patients, and although 16 died (mean survival, 5 months), 5 were still alive at the time the paper was submitted, with the two longest survivors having survived for 10 years. In the past 2 years, five small retrospective reports have reported on a total of 102 patients undergoing hepatectomy, with 5year survivals between $20 \%$ and $38 \%$ [49-53], suggesting that liver resection could be considered in patients in whom this could result in an $\mathrm{R} 0$ resection.

Although the proportion of recurrences that are hepatic is considerable (14\% to $33 \%$ ) [11,19], the proportion of the total number of patients undergoing curative resection who develop hepatic secondaries is small, and even fewer are suitable for resection. Okano et al. [53] reported that, between 1986 and 1999, of 807 patients undergoing surgery for gastric cancer, only 12 cases
$(1.5 \%)$ of hepatic recurrences were found, and only 6 $(0.7 \%)$ of these were suitable for resection. Likewise, Sakamoto et al. [50] reported that, of 4730 patients undergoing gastrectomy between 1985 and 2001, 122 (3\%) were found to have hepatic recurrence, but only $10(0.2 \%)$ were suitable for resection. Although the number of patients in each series is small, several of the studies reported more favorable survival in patients with single metastatic lesions $[48-50,53]$ and poorer survival where venous or lymphatic invasion was a feature of the original tumor $[47,49]$.

With the possible exception of a few hepatic metastases, the vast majority of recurrences are not curable, and any resection is likely to be palliative. In advanced primary gastric cancer such resections are associated with a high mortality and little survival benefit $[54,55]$. It is unlikely that patients with recurrence will fare any better, and most surgeons would reserve palliative surgery for the relief of obstructive symptoms.

\section{Chemotherapy}

The history of the development of chemotherapy for advanced gastric cancer is one of small phase II studies with promising response rates and large phase III studies demonstrating no improvement in survival [56-60]. Standing out among these negative trials are three RCTs that do demonstrate a significant survival benefit. The first, in 1984, demonstrated a benefit for 5fluorouracil (5-FU) and adriamycin plus mitomycin C (FAM) [61]. The second, in 1991, with 213 patients, demonstrated a benefit of methotrexate and 5-FU plus adriamycin (FAMTX) compared with FAM [56]. In the third trial, in 1997, epirubicin, cisplatin, and 5-FU (ECF) was shown to be superior to FAMTX [62]. Although these trials are encouraging, they should be taken in context, as the best median survival was in the 1991 trial with FAMTX, and this was only 42 weeks. In a subsequent RCT including FAMTX, it did not fare as well, with a median survival of only 30 weeks and a response rate of only $12 \%$ compared with $41 \%$ in the earlier trial [58]. Although the improvements in survival reported in these three trials were statistically significant, they were small, and the potential benefit appears in the improvement in 1- and 2-year survivals [61,63].

In many centers, chemotherapy has become the standard of care for advanced and recurrent gastric cancer and it is offered in the hope of improving survival and quality of life. It is not, however, universally seen to be beneficial, and its detractors point out that the number of negative RCTs far exceeds the number of positive trials. In the context of aggressive follow-up to detect asymptomatic disease, there is no evidence that treatment at an earlier stage improves outcomes, and in two studies of intensive follow-up, chemotherapy did not 
improve survival in the group with recurrences detected prior to their becoming symptomatic $[22,42]$.

For the alleviation of symptoms, response rates can - and do - translate into lessening symptoms and improved quality of life, but these patients, by their very nature, are not asymptomatic and are not helped by protocols designed to detect asymptomatic disease.

\section{Results of follow-up}

The literature regarding the benefits of intensive followup for gastric cancer is scarce. There are no RCTs and most reports are retrospective or observational. Very few report anything other than the detection of recurrences or death as the primary endpoints, and the implication is that recurrence equates to death. We discovered two studies on the efficacy of intensive follow-up, and both reported negatively. In the study by Kodera et al. [22], there were 88 patients detected with asymptomatic disease and 109 who presented symptomatically. Recurrences that were asymptomatic were more likely to be liver or distant metastases. More patients with asymptomatic disease underwent chemotherapy ( $88 \%$ vs $72 \%$ ) and resection of metastasis ( $10 \mathrm{vs}$ 5 ), but neither of these differences was significant. No patients were cured and there was no improvement in overall survival from the time of the original operation [22]. Bohner et al. [42] also found no benefit to intensive follow-up, although in their group, only 15 of the 67 recurrences were detected at an asymptomatic stage. Examining the observational studies, in the review of Sano et al. [20], of 1475 patients with EGC, all 20 patients with recurrence died. In the study of Yoo et al. [18], of 508 patients with recurrences, 48 (9\%) underwent further resection, but only $19(3.7 \%)$ were with curative intent. Of the 19 , only $5(1 \%)$ remained disease-free at the time of publication, and it is possible that they will suffer further recurrence [18].

\section{Discussion}

All surgeons follow up their patients, and some go to great efforts to detect recurrent disease, with the perceived benefit of increasing their patients' prospects of further curative treatment or at least an extension of life. Efforts at detecting recurrences early are fraught with difficulties. Imaging has relatively low sensitivity and the reports on the sensitivity of tumor markers vary. Even with intensive follow up, most recurrences are not detected until they become symptomatic $[22,42]$. The evidence that early detection of recurrent disease will lead to improved survival is also lacking. The nature of gastric cancer is that most recurrences are incurable by surgery at whatever stage they present [11,18], and the survival benefits of chemotherapy are small $[58,62,64]$. Despite theoretical advantages, there are no reports in the literature demonstrating benefit for earlier delivery of chemotherapy, but there are studies that demonstrate no survival benefit in patients treated for asymptomatic recurrences $[22,42]$.

There are, fortunately, some developments in the treatment of recurrent disease that may make a difference in future. New chemotherapeutic agents such as paclitaxel, CPT-11, and S-1 are yielding higher response rates and these may translate to improved survival. The results of three large ongoing Japanese RCTs of S-1, perhaps the most promising agent, are expected to be reported in 2006. On the surgical front, liver resection might become as acceptable for gastric cancer as it is for colorectal cancer. Between $1.5 \%$ and $3 \%$ of gastrectomy patients will develop hepatic metastasis, and of these, between $8 \%$ and $50 \%$ are potentially resectable, with reported 5 -year survival rates of between $20 \%$ and $38 \%[50,52,53]$. However, the nature of early reports is that they invariably overestimate survival advantages, and until a large prospective study has demonstrated benefit, liver resection cannot be assumed to be a standard form of treatment.

Intensive follow-up has the potential to detect new gastric cancers in the remnant stomach, and these cancers are often amenable to treatment [28-30]. However, after gastrectomy for benign disease, the incidence of second primaries is low, comparable to that of primary gastric cancers [26,29], and at least one nonrandomized trial has demonstrated no benefit from endoscopic surveillance in this group [65].

In two large studies of EGC, the death rates from nongastric cancers were significantly higher than those for recurrent or new gastric cancers [20,21]. The validity of what is effectively screening patients for other cancers is dependent on national priorities. In wealthy countries where population screening is the norm it may be justified, but in less wealthy countries without such programs, follow-up for these reasons might appear inappropriate.

It has been argued that follow-up may benefit patients' psychological wellbeing. While it is true that negative investigations can be reassuring, there is no evidence that complex investigations have any more psychological benefit than simple tests. Patients believe that negative tests mean that they are cancer-free and that that the detection of early recurrence, like early detection of their original cancer, will give them a chance of curative treatment. Intensive investigations by physicians reinforce these false beliefs. A patient's psychological wellbeing can be shattered by positive tests. If truly indicative of a recurrence, the knowledge that a cure is not possible leaves a permanent shadow 


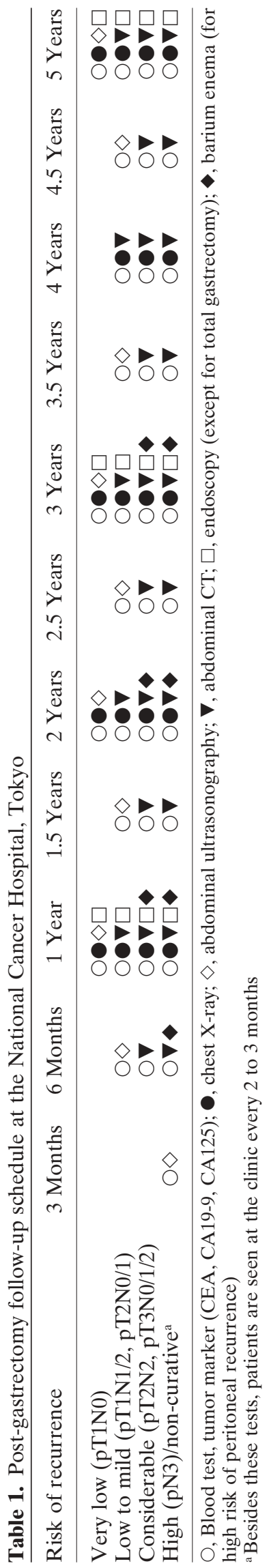

over the remains of the patients' life. If the test is a falsepositive, it can be very difficult to refute and the uncertainty of diagnosis invokes very significant anxiety.

Patient follow-up protocols vary widely [9]. Table 1 shows the follow-up protocol for the NCC, Tokyo. The large numbers of patients undergoing follow-up at the NCC and the recognition of very low recurrence rates for node-negative EGC has led to the routine use of follow-up CT for these patients being abandoned. This is not commonly the case elsewhere in Japan, and many patients have multiple CT scans annually.

In 1996 in Japan there were 103000 cases of gastric cancer, and in 1999, 50500 deaths [66]. The number of patients that would be under follow-up if patients were discharged after 5 years would be in the order of 250000. If patients were followed for 10 years it would be nearly 400000 . As well as cost and resource implications, follow-up CT scanning of these patients annually or more frequently results in a considerable cumulative exposure to radiation. A recent publication in the Lancet has estimated that $3 \%$ of all cancers in Japan are the result of radiation from diagnostic imaging [67]. Potentially, follow-up could cause more deaths than lives saved.

Follow-up is important. Collection of data to assess outcomes of treatment is vital if the quality of treatments is to be improved, and there is little doubt that many patients enjoy seeing their doctors. We should, however, be aware of the limitations of follow-up and that there is a huge amount of time and money being spent on investigations with little evidence of benefit. In these days of evidence-based medicine and stringent financial controls, what is required is a large, prospective RCT to determine what the benefits of intensive follow-up and early treatment of recurrence actually are, and whether they are worthwhile pursuing.

Acknowledgments This work was partly supported by the Second Term Comprehensive 10-Year Strategy for Cancer Control by the Ministry of Health and Welfare, Japan. The authors are grateful for the support of the Foundation for Promotion of Cancer Research, Japan.

\section{References}

1. Hallissey MT, Allum WH, Jewkes AJ, Ellis DJ, Fielding JW. Early detection of gastric cancer. BMJ 1990;301:513-5.

2. Sue-Ling HM, Johnston D, Martin IG, Dixon MF, Lansdown MR, McMahon MJ, et al. Gastric cancer: a curable disease in Britain. BMJ 1993;307:591-6.

3. Renehan AG, Egger M, Saunders MP, O'Dwyer ST. Impact on survival of intensive follow up after curative resection for colorectal cancer: systematic review and meta-analysis of randomized trials. BMJ 2002;324:813-6.

4. Guidelines for the management of colorectal cancer. www.asgbi.org.uk 2001 cited; available from: www.asgbi.org.uk 
5. Desch CE, Benson AB 3rd, Smith TJ, Flynn PJ, Krause C, Loprinzi CL, et al. Recommended colorectal cancer surveillance guidelines by the American Society of Clinical Oncology. J Clin Oncol 1999;17:1312-21.

6. Smith TJ, Davidson NE, Schapira DV, Grunfeld E, Muss HB, Vogel VG 3rd, et al. American Society of Clinical Oncology 1998 update of recommended breast cancer surveillance guidelines. J Clin Oncol 1999;17:1080-2.

7. Clinical practice guidelines for the treatment of unresectable nonsmall-cell lung cancer. Adopted on May 16, 1997 by the American Society of Clinical Oncology. J Clin Oncol 1997;15:2996-3018.

8. Nakajima T. Gastric cancer treatment guidelines in Japan. Gastric Cancer 2002;5:1-5.

9. Johnson FE, Virgo KS. Cancer patient follow-up. St Louis: Mosby; 1997.

10. Roviello F, Marrelli D, de Manzoni G, Morgagni P, Di Leo A, Saragoni L, et al. Prospective study of peritoneal recurrence after curative surgery for gastric cancer. Br J Surg 2003;90:11139.

11. Maehara Y, Hasuda S, Koga T, Tokunaga E, Kakeji Y, Sugimachi K. Postoperative outcome and sites of recurrence in patients following curative resection of gastric cancer. Br J Surg. 2000;87:3537.

12. Shiraishi N, Inomata M, Osawa N, Yasuda K, Adachi Y, Kitano S. Early and late recurrence after gastrectomy for gastric carcinoma. Univariate and multivariate analyses. Cancer 2000;89:255-61.

13. Ohno S, Fujii T, Ueda S, Nakamoto T, Kinugasa S, Yoshimura H, et al. Predictive factors and timing for liver recurrence after curative resection of gastric carcinoma. Am J Surg 2003;185:258-63.

14. Schwarz RE, Zagala-Nevarez K. Recurrence patterns after radical gastrectomy for gastric cancer: prognostic factors and implications for postoperative adjuvant therapy. Ann Surg Oncol 2002;9:394-400.

15. Arima N, Adachi K, Katsube T, Amano K, Ishihara S, Watanabe $\mathrm{M}$, et al. Predictive factors for metachronous recurrence of early gastric cancer after endoscopic treatment (see comment). J Clin Gastroenterol 1999;29:44-7.

16. Uemura N, Mukai T, Okamoto S, Yamaguchi S, Mashiba H, Taniyama K, et al. Effect of Helicobacter pylori eradication on subsequent development of cancer after endoscopic resection of early gastric cancer. Cancer Epidemiol Biomarkers Prev 1997; 6:639-42.

17. Gunderson LL, Sosin H. Adenocarcinoma of the stomach: areas of failure in a re-operation series (second or symptomatic look) clinicopathologic correlation and implications for adjuvant therapy. Int J Radiat Oncol Biol Phy 1982;8:1-11.

18. Yoo CH, Noh SH, Shin DW, Choi SH, Min JS. Recurrence following curative resection for gastric carcinoma. Br J Surg 2000;87: 236-42.

19. Katai H, Maruyama K, Sasako M, Sano T, Okajima K, Kinoshita $\mathrm{T}$, et al. Mode of recurrence after gastric cancer surgery. Dig Surg 1994;11:99-103.

20. Sano T, Sasako M, Kinoshita T, Maruyama K. Recurrence of early gastric cancer. Follow-up of 1475 patients and review of the Japanese literature (see comment). Cancer 1993;72:3174-8.

21. Lee HJ, Kim YH, Kim WH, Lee KU, Choe KJ, Kim JP, et al. Clinicopathological analysis for recurrence of early gastric cancer. Jpn J Clin Oncol 2003;33:209-14.

22. Kodera Y, Ito S, Yamamura Y, Mochizuki Y, Fujiwara M, Hibi K, et al. Follow-up surveillance for recurrence after curative gastric cancer surgery lacks survival benefit. Ann Surg Oncol 2003;10: 898-902.

23. Macdonald JS, Smalley SR, Benedetti J, Hundahl SA, Estes NC, Stemmermann GN, et al. Chemoradiotherapy after surgery compared with surgery alone for adenocarcinoma of the stomach or gastroesophageal junction (see comment). New Engl J Med 2001:345:725-30.

24. Hironaka SI, Boku N, Ohtsu A, Nagashima F, Sano Y, Muto M, et al. Sequential methotrexate and 5-fluorouracil therapy for gas- tric cancer patients with bone metastasis. Gastric Cancer 2000;3: 19-23.

25. Tersmette AC, Offerhaus GJ, Tersmette KW, Giardiello FM, Moore GW, Tytgat GN, et al. Meta-analysis of the risk of gastric stump cancer: detection of high risk patient subsets for stomach cancer after remote partial gastrectomy for benign conditions. Cancer Res 1990;50:6486-9.

26. Lundegardh G, Adami HO, Helmick C, Zack M, Meirik O. Stomach cancer after partial gastrectomy for benign ulcer disease. N Engl J Med 1988;319:195-200.

27. Kikuchi S, Sato M, Katada N, Sakuramoto S, Shimao H, Kakita A, et al. Efficacy of endoscopic surveillance of the upper gastrointestinal tract following distal gastrectomy for early gastric cancer. Hepatogastroenterology 2003;50:1704-7.

28. Thorban S, Bottcher K, Etter M, Roder JD, Busch R, Siewert JR. Prognostic factors in gastric stump carcinoma. Ann Surg 2000;231: 188-94.

29. Han SL, Hua YW, Wang CH, Ji SQ, Zhuang J. Metastatic pattern of lymph node and surgery for gastric stump cancer. J Surg Oncol 2003;82:241-6.

30. Sasako M, Maruyama K, Kinoshita T, Okabayashi K. Surgical treatment of carcinoma of the gastric stump. Br J Surg 1991;78: $822-4$.

31. Chen CN, Lee WJ, Lee PH, Chang KJ, Chen KM. Clinicopathologic characteristics and prognosis of gastric stump cancer. J Clin Gastroenterol 1996;23:251-5.

32. Tas F, Faruk Aykan N, Aydiner A, Yasasever V, Topuz E. Measurement of serum CA 19-9 may be more valuable than CEA in prediction of recurrence in patients with gastric cancer. Am J Clin Oncol 2001;24:148-9.

33. Lai IR, Lee WJ, Huang MT, Lin HH. Comparison of serum CA72-4, CEA, TPA, CA19-9 and CA125 levels in gastric cancer patients and correlation with recurrence. Hepatogastroenterology 2002;49:1157-60.

34. Takahashi Y, Takeuchi T, Sakamoto J, Touge T, Mai M, Ohkura $\mathrm{H}$, et al. The usefulness of CEA and/or CA19-9 in monitoring for recurrence in gastric cancer patients: a prospective clinical study. Gastric Cancer 2003;6:142-5.

35. Ikeda Y, Mori M, Kajiyama K, Kamakura T, Maehara Y, Haraguchi $\mathrm{Y}$, et al. Indicative value of carcinoembryonic antigen (CEA) for liver recurrence following curative resection of stage II and III gastric cancer. Hepatogastroenterology 1996;43:12817.

36. Ha HK, Kim HH, Kim HS, Lee MH, Kim KT, Shinn KS. Local recurrence after surgery for gastric carcinoma: CT findings. AJR Am J Roentgenol 1993;161:975-7.

37. Sano T, Yamamoto S, Sasako M. Randomized controlled trial to evaluate splenectomy in total gastrectomy for proximal gastric carcinoma: Japan Clinical Oncology Group study JCOG 0110MF. Jpn J Clin Oncol 2002;32:363-4.

38. Kinkel K, Lu Y, Both M, Warren RS, Thoeni RF. Detection of hepatic metastases from cancers of the gastrointestinal tract by using noninvasive imaging methods (US, CT, MR imaging, PET): a meta-analysis. Radiology 2002;224:748-56.

39. Insko EK, Levine MS, Birnbaum BA, Jacobs JE. Benign and malignant lesions of the stomach: evaluation of CT criteria for differentiation. Radiology 2003;228:166-71.

40. Meyers MA, McSweeney J. Secondary neoplasms of the bowel. Radiology 1972;105:1-11.

41. Jadvar H, Tatlidil R, Garcia AA, Conti PS. Evaluation of recurrent gastric malignancy with [F-18]-FDG positron emission tomography (erratum appears in Clin Radiol 2003 Jul;58:570). Clin Radiol 2003;58:215-21.

42. Bohner H, Zimmer T, Hopfenmuller W, Berger G, Buhr HJ. Detection and prognosis of recurrent gastric cancer - is routine follow-up after gastrectomy worthwhile? Hepatogastroenterology. 2000;47:1489-94.

43. Inada T, Ogata Y, Andoh J, Ozawa I, Matsui J, Hishinuma S, et al. Significance of para-aortic lymph node dissection in patients with 
advanced and recurrent gastric cancer. Anticancer Res 1994;14: 677-82.

44. Takeyoshi I, Ohwada S, Ogawa T, Kawashima Y, Ohya T, Kawate $\mathrm{S}$, et al. The resection of non-hepatic intraabdominal recurrence of gastric cancer. Hepatogastroenterology 2000;47: 1479-81.

45. Choti MA, Sitzmann JV, Tiburi MF, Sumetchotimetha W, Rangsin R, Schulick RD, et al. Trends in long-term survival following liver resection for hepatic colorectal metastases. Ann Surg 2002;235:759-66.

46. Minagawa M, Makuuchi M, Torzilli G, Takayama T, Kawasaki S, Kosuge T, et al. Extension of the frontiers of surgical indications in the treatment of liver metastases from colorectal cancer: longterm results. Ann Surg 2000;231:487-99.

47. Ochiai T, Sasako M, Mizuno S, Kinoshita T, Takayama T, Kosuge $\mathrm{T}$, et al. Hepatic resection for metastatic tumours from gastric cancer: analysis of prognostic factors. Br J Surg 1994;81:1175-8.

48. Miyazaki M, Itoh H, Nakagawa K, Ambiru S, Shimizu H, Togawa A, et al. Hepatic resection of liver metastases from gastric carcinoma. Am J Gastroenterol 1997;92:490-3.

49. Shirabe K, Shimada M, Matsumata T, Higashi H, Yakeishi Y, Wakiyama S, et al. Analysis of the prognostic factors for liver metastasis of gastric cancer after hepatic resection: a multiinstitutional study of the indications for resection. Hepatogastroenterology 2003;50:1560-3.

50. Sakamoto Y, Ohyama S, Yamamoto J, Yamada K, Seki M, Ohta $\mathrm{K}$, et al. Surgical resection of liver metastases of gastric cancer: an analysis of a 17-year experience with 22 patients. Surgery 2003; 133:507-11.

51. Zacherl J, Zacherl M, Scheuba C, Steininger R, Wenzl E, Muhlbacher F, et al. Analysis of hepatic resection of metastasis originating from gastric adenocarcinoma. J Gastrointest Surg 2002;6:682-9.

52. Saiura A, Umekita N, Inoue S, Maeshiro T, Miyamoto S, Matsui $\mathrm{Y}$, et al. Clinicopathological features and outcome of hepatic resection for liver metastasis from gastric cancer. Hepatogastroenterology 2002;49:1062-5.

53. Okano K, Maeba T, Ishimura K, Karasawa Y, Goda F, Wakabayashi $\mathrm{H}$, et al. Hepatic resection for metastatic tumors from gastric cancer. Ann Surg 2002;235:86-91.

54. Hartgrink HH, Putter H, Klein Kranenbarg E, Bonenkamp JJ, van de Velde CJ. Value of palliative resection in gastric cancer. Br J Surg 2002;89:1438-43.

55. Haugstvedt T, Viste A, Eide GE, Soreide O. The survival benefit of resection in patients with advanced stomach cancer: the Norwegian multicenter experience. Norwegian Stomach Cancer Trial. World J Surg 1989;13:617-21; discussion 621-2.

56. Wils JA, Klein HO, Wagener DJ, Bleiberg H, Reis H, Korsten F, et al. Sequential high-dose methotrexate and fluorouracil combined with doxorubicin - a step ahead in the treatment of advanced gastric cancer: a trial of the European Organization for Research and Treatment of Cancer Gastrointestinal Tract Cooperative Group. J Clin Oncol 1991;9:827-31.

57. Murad AM, Santiago FF, Petroianu A, Rocha PR, Rodrigues MA, Rausch M. Modified therapy with 5-fluorouracil, doxorubicin, and methotrexate in advanced gastric cancer. Cancer 1993;72: 37-41.

58. Vanhoefer U, Rougier P, Wilke H, Ducreux MP, Lacave AJ, Van Cutsem E, et al. Final results of a randomized phase III trial of sequential high-dose methotrexate, fluorouracil, and doxorubicin versus etoposide, leucovorin, and fluorouracil versus infusional fluorouracil and cisplatin in advanced gastric cancer: a trial of the European Organization for Research and Treatment of Cancer Gastrointestinal Tract Cancer Cooperative Group (comment). J Clin Oncol 2000;18:2648-57.

59. Moynihan T, Hansen R, Anderson T, Quebbeman E, Beatty P, Ausman R, et al. Continuous 5-fluorouracil infusion in advanced gastric carcinoma. Am J Clin Oncol 1988;11:461-4.

60. Ohtsu A, Shimada Y, Shirao K, Boku N, Hyodo I, Saito H, et al. Randomized phase III trial of fluorouracil alone versus fluorouracil plus cisplatin versus uracil and tegafur plus mitomycin in patients with unresectable, advanced gastric cancer: The Japan Clinical Oncology Group Study (JCOG9205). J Clin Oncol 2003; 21:54-9.

61. Douglass HO Jr, Lavin PT, Goudsmit A, Klaassen DJ, Paul AR. An Eastern Cooperative Oncology Group evaluation of combinations of methyl-CCNU, mitomycin $\mathrm{C}$, adriamycin, and 5-fluorouracil in advanced measurable gastric cancer (EST 2277). J Clin Oncol 1984;2:1372-81.

62. Webb A, Cunningham D, Scarffe JH, Harper P, Norman A, Joffe $\mathrm{JK}$, et al. Randomized trial comparing epirubicin, cisplatin, and fluorouracil versus fluorouracil, doxorubicin, and methotrexate in advanced esophagogastric cancer. J Clin Oncol 1997;15: 261-7.

63. Waters JS, Norman A, Cunningham D, Scarffe JH, Webb A, Harper $\mathrm{P}$, et al. Long-term survival after epirubicin, cisplatin and fluorouracil for gastric cancer: results of a randomized trial. Br J Cancer 1999;80:269-72.

64. Wils J, Bleiberg H, Dalesio O, Blijham G, Mulder N, Planting A, et al. An EORTC Gastrointestinal Group evaluation of the combination of sequential methotrexate and 5-fluorouracil, combined with adriamycin in advanced measurable gastric cancer. J Clin Oncol 1986;4:1799-803.

65. Stael von Holstein C, Eriksson S, Huldt B, Hammar E. Endoscopic screening during 17 years for gastric stump carcinoma. A prospective clinical trial. Scand J Gastroenterol 1991;26:10206.

66. Cancer Statistics in Japan. (Cited; available from: http://www. ncc.go.jp/en/statistics/index.html)

67. Berrington de Gonzalez A, Darby S. Risk of cancer from diagnostic X-rays: estimates for the United Kingdom and 14 other countries. Lancet 2004;363:345-51. 